\title{
Hydrodynamical models for low-luminosity GRBs and relativistic $\mathrm{SNe}$
}

\author{
Akihiro Suzuki* \\ Department of Astronomy, Kyoto University \\ E-mail: asuzukidkusastro.kyoto-u.ac.jp \\ Keiichi Maeda \\ Department of Astronomy, Kyoto University ${ }^{\dagger}$

\section{Toshikazu Shigeyama} \\ Research Center for the Early Universe, School of Science, University of Tokyo
}

\begin{abstract}
We present results of two-dimensional special relativistic hydrodynamical simulations of GRB jet propagation in a massive star and explosive nucleosynthesis taking place in the star as a result of the jet injection. We consider jets with various energy deposition rates and succeed in reproducing both ultra-relativistic jet and failed jet cases. Then, we perform calculations of explosive nucleosynthesis by solving a nuclear reaction network for the thermal history of tracer particles. From the thus obtained chemical composition of ejecta realized in different jet models, we specify some nuclei, such as, ${ }^{56} \mathrm{Ni}$ and ${ }^{4} \mathrm{He}$, characterizing the jet injection condition. Our results indicate that comparison of the calculated chemical composition with spectroscopic observations of $\mathrm{SNe}$ with and without GRBs provides us important clues toward understanding the nature of jet-driven explosions.
\end{abstract}

Swift: 10 Years of Discovery,

2-5 December 2014

La Sapienza University, Rome, Italy

\footnotetext{
* Speaker.

†also at Kavli Institute for the Physics and Mathematics of the Universe (WPI), Todai Institutes for Advanced Study, University of Tokyo
} 


\section{Introduction}

It is now widely known that long-duration gamma-ray bursts (long GRBs) are closely linked to the death of massive stars (see, e.g., Refs. [四, ]. for reviews). In some long GRBs found in the nearby universe, a bump is found in their afterglow light curves and its spectrum well agrees with those of a specific class of supernovae (SNe), broad-lined Ic SNe. Although there are only handful numbers of GRBs with spectroscopically identified SNe, significant fraction of these events shows gamma-ray isotropic luminosity smaller than those of standard GRBs found at cosmological distances. These events are called low-luminosity GRBs (LLGRBs). For example, GRBs 980425, 060218, and 100316D, which were associated with SNe 1998bw, 2006aj, and 2010bh, are classified as LLGRBs. Some questions regarding these events, e.g., what is the mechanism giving rise to their X-ray and gamma-ray emission and what makes the difference between standard and lowluminosity GRBs, remain under debate.

It has been claimed that they are a distinct class of gamma-ray burst. First, the volumetric rate of LLGRBs appears to be larger than that of standard GRBs $\left(\sim 1 \mathrm{Gpc}^{-3} \mathrm{yr}^{-1}\right.$, e.g., [目]). So far, several authors have estimated the volumetric rate and, for example, the following estimated values, $380_{-225}^{+620} \mathrm{Gpc}^{-3} \mathrm{yr}^{-1}$ by Ref. [四] and $325_{-177}^{+352} \mathrm{Gpc}^{-3} \mathrm{yr}^{-1}$ by Ref. [[5], have been reported. These values suggest that one cannot explain them as the lower end of the luminosity function of standard GRBs, supporting their distinct origin. Recently, in Refs. [ [6, 四], Bromberg et al. developed an analytical model for the jet propagation in massive stars and applied their model to LLGRBs. They pointed out that the duration of the energy injection seems to be shorter for LLGRBs than the time of the jet penetration and thus the jet should fail to emerge from the star. Numerical simulations performed in Ref [ [8] reproduced the failed jet .

In this study, we carry out numerical simulations of the jet propagation in a massive star for a various set of the jet injection condition to study the ejecta resulting from the jet injection. The jet injection produce a shocked region in the star, in which stellar mantles undergo explosive nucleosynthesis. From the thermal history of tracer particles, we solve the nuclear reaction network to obtain the chemical abundance of the ejecta. In Section 2, we briefly describe our method of the calculations. Results of the calculations are presented in Section 3. Section 4 concludes this paper.

\section{Method}

We carry out simulations of a GRB jet penetrating a massive star by using a numerical code developed by one of the authors [Q]. We use one of the pre-supernova models provided by Woosley $\&$ Heger [प]], the model 16TI, as the progenitor star. The star has a radius of $4 \times 10^{10} \mathrm{~cm}$ and a mass of $14 M_{\odot}$ prior to the collapse of the iron core. We inject a relativistic jet with a fixed total energy and $5 \times 10^{52}$ erg and various energy deposition rates at the injection radius $R_{\text {in }}$ in a similar way to the previous works (e.g., Ref. [प]]). The Lorentz factor, specific enthalpy, and the half opening angle of the jet are also fixed to be $\Gamma_{\mathrm{j}}=5, \varepsilon_{0} / c^{2}=20$, and $\theta_{\mathrm{j}}=10^{\circ}$. We perform simulations with the energy deposition rates of $\dot{E}_{50}=2000,1000,500,200,100,50,20,10$, and 5, where $\dot{E}_{50}$ denotes the energy deposition rate in units of $10^{50} \mathrm{erg} \mathrm{s}^{-1}$, and the injection radii of $R_{\text {in }}=1.5 \times 10^{8}$ and $10^{9}$ $\mathrm{cm}$. The chemical composition of the ejecta is obtained by a post-process calculation. The thermal history of 16384 tracer particles, which are located in the star at $t=0$ and move along the velocity 

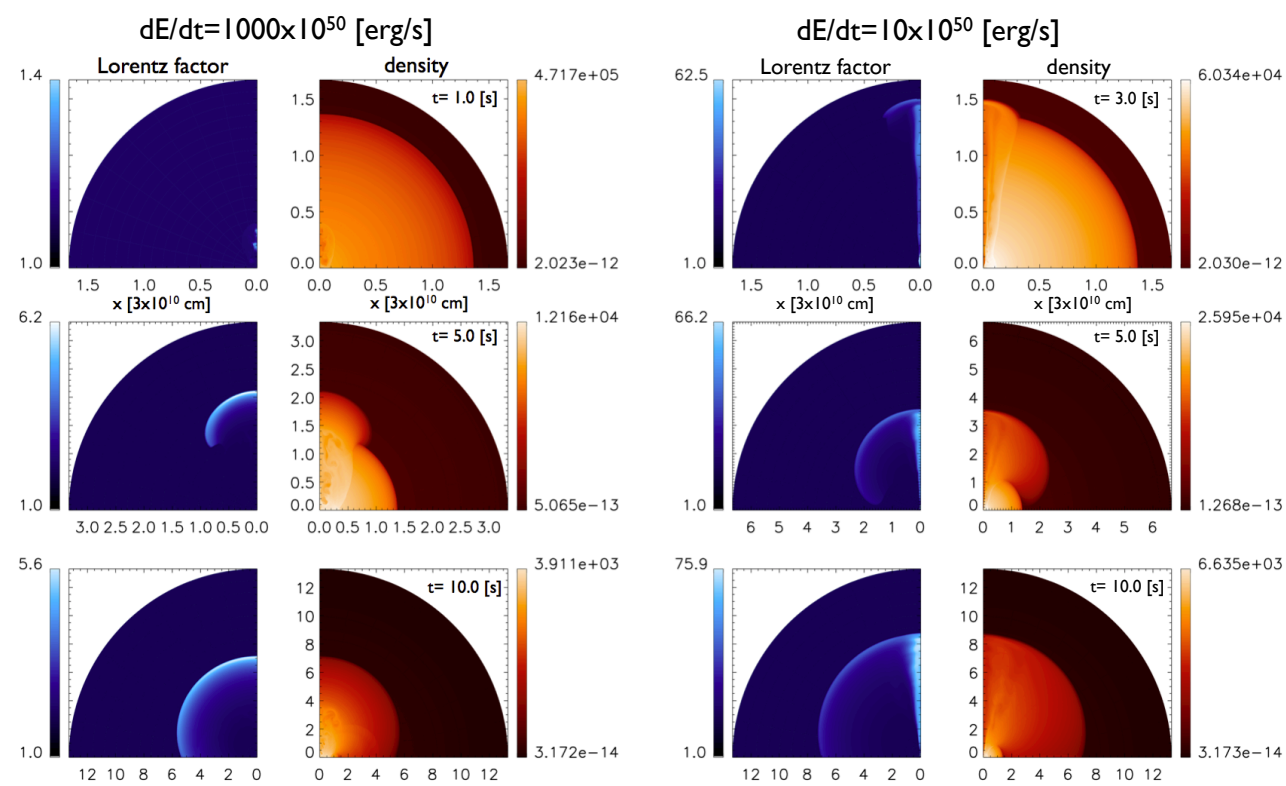

Figure 1: Spatial distributions of the Lorentz factor and the density for the model with $\dot{E}_{50}=1000$ and $R_{\text {in }}=3 \times 10^{8} \mathrm{~cm}$ at $t=1.0$ (top), 5.0 (middle), and 10.0 (bottom) s after the jet injection.

field obtained in the simulation, is used to solve the nuclear reaction networks composed of 490

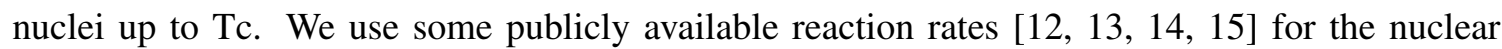
reaction network.

\section{Results}

Figure $\square$ shows the temporal evolution of the jet for the models with $\dot{E}_{50}=1000$ (left) and 10 (right) and with the injection radius of $R_{\mathrm{in}}=3 \times 10^{8} \mathrm{~cm}$. The top, middle, and bottom panels show the snapshots of the spatial distributions of the Lorentz factor (left) and the the density (right) at different epochs. In the model with $\dot{E}_{50}=1000$, the jet injection time $E_{\text {tot }} /(2 \dot{E})$ is shorter than the time required for the jet to penetrate the star, resulting in quasi-spherical ejecta expanding at mildly relativistic speeds. On the other hand, for models with low energy deposition rates, the jet injection time is sufficiently long to penetrate the star. After the penetration, the jet can escape from the star without dissipating its energy, resulting in a collimated jet with a Lorentz factor lager than 100.

The kinetic energy distribution of ejecta defined as follows,

$$
E_{\mathrm{k}}(>\Gamma \beta)=\int_{>\Gamma \beta} \Gamma(\Gamma-1) \rho c^{2} d V
$$

where the integration is performed for the ejecta moving at 4-velocities faster than $\Gamma \beta$, is often used to specify the properties of the ejecta (e.g., Ref. [ए6]). This quantity is calculated for our models and the results are presented in Figure $\square$. The left and right panels of Figure $\square$ correspond to the models with the smaller and larger injection radii. In Ref. [ㅁ]], Tan et al. calculated the kinetic energy distribution of trans-relativistic ejecta resulting from a spherical explosion. They found that 

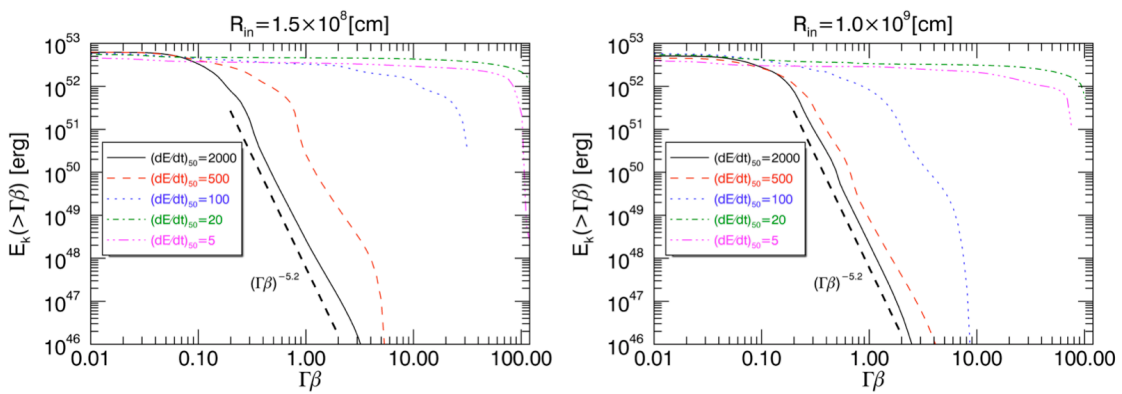

Figure 2: Kinetic energy distributions of ejecta at $t=100 \mathrm{~s}$ in our hydrodynamical simulations.

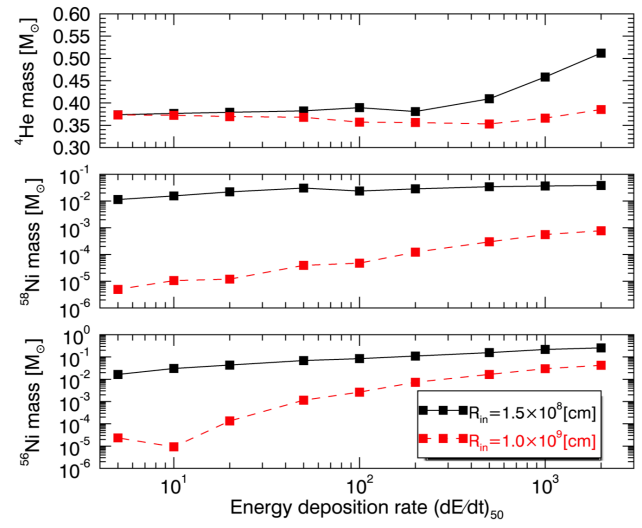

Figure 3: Mass of some elements in the ejecta as functions of the energy deposition rate.

the distribution can be represented by a power-law distribution with an index -5.2 , which is also shown as a thick dashed line in the panels in Figure [ 2 . The models with higher energy deposition rates clearly reproduce kinetic energy distributions similar to the spherical case. As shown in the

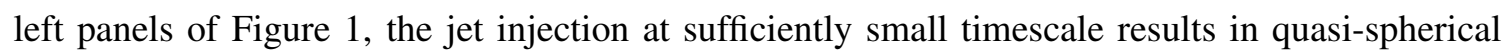
ejecta. This is why the kinetic energy distribution of this model well agree with the spherical case. On the other hand, for the models with high energy deposition rates, the kinetic distribution exhibit very shallow slope, which means that a considerable amount of the energy is carried by ejecta moving at highly relativistic speeds. As shown in the right panel of Figure $\mathbb{W}$, an ultra-relativistic jet is realized as a result of the jet injection at a lower energy injection rate, which corresponds to the highly relativistic part of the kinetic energy distribution.

In Figure B], we show the masses of ${ }^{56} \mathrm{Ni},{ }^{58} \mathrm{Ni}$, and ${ }^{4} \mathrm{He}$ in the ejecta, as functions of the energy deposition rate. The radioactive decay of ${ }^{56} \mathrm{Ni}$ can serve as an energy source of the ejecta, creating a SN bump at a few days to several 10 days after the explosion. The amount of ${ }^{56} \mathrm{Ni}$ estimated from observations is of the order of $\sim 0.1 M_{\odot}$ for SNe associated with GRBs. The estimated amount is achieved only for models with high energy deposition rates and the smaller injection radius. 
Therefore, models reproducing a highly relativistic jet cannot produce sufficient amount of ${ }^{56} \mathrm{Ni}$, suggesting that another origin of ${ }^{56} \mathrm{Ni}$ is needed. Stable ${ }^{58} \mathrm{Ni}$ is found to be significantly sensitive to the injection radius as shown in Figure B. This is because neutron-rich materials in the vicinity of the iron core in the progenitor star can be mixed to the ejecta as a result of the jet injection in models with the smaller injection radius. In models with the larger injection radius, such neutronrich materials are assumed to fall into the central compact object. For models with higher energy deposition rates, the shocked gas can reach higher temperature than models with lower energy deposition rates and undergoes $\alpha$-rich freeze-out, through which ${ }^{4} \mathrm{He}$ is efficiently produced. The enhancement of ${ }^{4} \mathrm{He}$ is recognized in models with $\dot{E}_{50}>200$ and the smaller injection radius. Since the progenitor model contains $\sim 0.36 M_{\odot}$ of ${ }^{4} \mathrm{He}$ in its outer layer, the newly synthesized ${ }^{4} \mathrm{He}$ via explosive nucleosynthesis is up to $\sim 0.2 M_{\odot}$, which is realized in the model with the highest energy deposition rate.

\section{Conclusion}

In this study, we investigate the propagation of a jet in a massive star for various sets of the injection condition and how the parameters specifying the jet affect the chemical composition of the ejecta. The jet successfully accelerates to ultra-relativistic velocities for lower energy deposition rates, while models with higher energy deposition rates make failed jets. These results agrees with earlier studied on the jet propagation in a massive star, such as, Ref. [[]]

We have performed explosive nucleosynthesis calculations by using a tracer particle method to obtain the chemical composition of the ejecta realized in our hydrodynamical simulations. We find that the amount of some chemical elements synthesized via explosive nucleosynthesis are sensitive to the jet injection condition and thus can be used as a probe to investigating the properties of the jet. Sufficient amount of radioactive ${ }^{56} \mathrm{Ni}$ can be produced only when a jet is injected at a high energy deposition rate at a smaller injection radius. The presence of ${ }^{58} \mathrm{Ni}$ in ejecta, which has been pointed out for GRB 060218 associated with SN 2006aj [ए8], might indicate the jet injection at a smaller radius and thus a smaller central compact object. In models with high energy deposition rates, the jet fails to penetrate the star and the most injected energy thermalizes in the star, resulting in shocked gas at higher temperature than that in models with low energy deposition rates. The shocked gas undergoes $\alpha$-rich freeze-out, leading to efficient production of ${ }^{4} \mathrm{He}$. In fact, some peculiar type Ic supernovae with radio brightness comparable to LLGRBs, such as SNe 2009bb [미, [20] and 2012ap [], [22, [23, [24], show spectral features that could be caused by ${ }^{4} \mathrm{He}$ in their infrared spectra. ${ }^{4} \mathrm{He}$ produced by $\alpha$-rich freezeout might contribute to these spectral features. Further observational and theoretical studies on these events will give us important clues on jetdriven explosions of massive stars.

\section{References}

[1] T. Piran, Gamma-ray bursts and the fireball model, Physics Reports 314 (1999) 575

[2] P. Mészáros, Theories of Gamma-Ray Bursts, ARA\&A 40 (2002) 137

[3] D. Wanderman, \& T. Piran, The luminosity function and the rate of Swift's gamma-ray bursts, MNRAS, 406 (2010) 1944 
[4] D. Guetta, \& M. Della Valle, On the Rates of Gamma-Ray Bursts and Type Ib/c Supernovae, ApJL 657 (2007) L73

[5] E. Liang, B. Zhang, F. Virgili, \& Z. G. Dai, Low-Luminosity Gamma-Ray Bursts as a Unique Population: Luminosity Function, Local Rate, and Beaming Factor, ApJ 662 (2007) 1111

[6] O. Bromberg, E. Nakar, \& T. Piran Are Low-luminosity Gamma-Ray Bursts Generated by Relativistic Jets?, ApJL 739 (2011) L55

[7] O. Bromberg, E. Nakar, T. Piran, \& R. Sari, The Propagation of Relativistic Jets in External Media, ApJ 740 (2011) 100

[8] D. Lazzati, B. J. Morsony, C. H. Blackwell, \& M. C. Begelman, Unifying the Zoo of Jet-driven Stellar Explosions, ApJ 750 (2012) 68

[9] A. Suzuki, \& T. Shigeyama, Early Thermal X-Ray Emission from Long Gamma-Ray Bursts and Their Circumstellar Environments, ApJ 764 (2013) L12

[10] S. E. Woosley, \& A. Heger, The Progenitor Stars of Gamma-Ray Bursts, ApJ 637 (2006) 914

[11] W. Zhang, S. E. Woosley, \& A. I. MacFadyen, Relativistic Jets in Collapsars ApJ 586 (2003) 356

[12] T. Rauscher, \& F.-K. Thielemann, Astrophysical Reaction Rates From Statistical Model Calculations, Atomic Data and Nuclear Data Tables 75 (2000) 1

[13] G. M. Fuller, W. A. Fowler, \& M. J. Newman, Stellar weak-interaction rates for sd-shell nuclei. I Nuclear matrix element systematics with application to Al-26 and selected nuclei of importance to the supernova problem, ApJS 42 (1980) 447

[14] G. M. Fuller, W. A. Fowler, \& M. J. Newman, Stellar weak interaction rates for intermediate-mass nuclei. II - A = 21 to $A=60$, ApJ 252 (1982) 715

[15] G. M. Fuller, W. A. Fowler, \& M. J. Newman, Stellar weak interaction rates for intermediate mass nuclei. III - Rate tables for the free nucleons and nuclei with A = 21 to A =60, ApJS 48 (1982) 279

[16] C. D. Matzner, \& C. F., McKee, The Expulsion of Stellar Envelopes in Core-Collapse Supernovae, ApJ 510 (1999) 379

[17] J. C. Tan, C. D. Matzner, \& C. F. McKee, Trans-Relativistic Blast Waves in Supernovae as Gamma-Ray Burst Progenitors, ApJ 551 (2001) 946

[18] K. Maeda, P. A. Mazzali, \& K. Nomoto, Optical Emission from Aspherical Supernovae and the Hypernova SN 1998bw, ApJ 645 (2006) 1331

[19] A. M. Soderberg, S. Chakraborti, G. Pignata, et al. A relativistic type Ibc supernova without a detected $\boldsymbol{r}$-ray burst, Nature 463 (2010) 513

[20] M. F. Bietenholz, A. M. Soderberg, N. Bartel, et al. VLBI Observations of the Type I b/c Supernova $2009 b b$, ApJ 725 (2010) 4

[21] D. Xu, J.-J. Zhang, J. Chen, et al. The Astronomer's Telegram 3922 (2012) 1

[22] D. Milisavljevic, R. Fesen, A. Soderberg, et al. Central Bureau Electronic Telegrams 3037 (2012) 2

[23] S. Chakraborti, A. Soderberg, L. Chomiuk, et al. A Missing-Link in the Supernova-GRB Connection: The Case of SN 2012ap, arXiv:1402.6336

[24] D. Milisavljevic, R. Margutti, J. T. Parrent, et al. The Broad-lined Type Ic SN 2012ap and the Nature of Relativistic Supernovae Lacking a Gamma-Ray Burst Detection, ApJ 799 (2015) 51 